\title{
Crystalline Silcion Carbide Y Junctions Induced by Catalysts Coalescence
}

\author{
Zhenyu Liu*, V. Srot ${ }^{* *}$, and Judith C. Yang ${ }^{* * *}$ \\ ${ }_{* *}^{*}$ Department of Mechanical Engineering and Materials Science, University of Pittsburgh, PA 15261 \\ ${ }^{* *}$ Max-Planck-Institut für Metallforschung, Heisenbergstrasse 3, D-70569 Stuttgart, Germany \\ ${ }^{* * *}$ Department of Chemical and Petroleum Engineering, University of Pittsburgh, PA 15261
}

Nanoscale materials are currently being exploited as active components in a wide range of technological applications in different fields, such as composite materials, chemical sensing, biomedicine, optoelectronics and nanoelectronics. Many recent efforts have focused on the creation of various nanostructures including spheres, tubes, cables, sheets and, especially, wires, have been successfully synthesized, since these nanoscale building blocks are anticipated to transform the semiconductor industry. ${ }^{1-2}$ However, the realization of practical nanodevices requires that these nanostructures be integrated efficiently and economically into various intricate architectures. Synthetic control from bottom-up approach of one-dimensional (1D) nanostructures into complex heterostructures would impact the design of future electronic devices by simplifying much of the processing associated with nanodevice fabrications.

Among the various semiconductors, silicon carbide $(\mathrm{SiC})$ is an important group IV-IV compound that possesses unique physical and electronic properties. SiC-based electronics will be better than silicon-based devices in certain applications, because they can operate at higher temperature, higher power, and higher frequency as well as withstand harsh environments but with reduced cooling requirements. Our previous results demonstrated that $\mathrm{SiC}$ nanocones and heterostructures can be created by utilizing iron nanoparticles, that are originally encapsulated in a graphite-like shell, to catalyze a vapor-solid reaction between carbon and silicon monoxide vapor. Herein, we present that crystalline SiC Y-junctions can be formed by the coalescence of $\mathrm{Fe}$ catalysts, which have already catalyzed the formation of a single $\mathrm{SiC}$ nanowire creating a $\mathrm{SiC} \mathrm{Y}$ junction with either parallel or inclined branches.

Two kinds of crystalline SiC Y junctions have been observed, from the systematic TEM observation, to show either two inclined branches or parallel branches. This variation in branching corresponds to the branch growth direction mismatch and growth without direction mismatching. Noting that the catalyst droplets are located at the tip of the stems, the branches must form prior to the stems. Therefore, the formations of the crystalline SiC Y junctions are attributed to the coalescence of two catalyst droplets that have already each formed a $\mathrm{SiC}$ nanowire branch, while the merged catalyst remains catalytically active and catalyzes the stem formation. Moreover, "V"-shaped SiC nanostructures are detected in the products, in which the catalyst is located at the intersection between two branches, which supports the catalyst droplets coalescence mechanism, as shown in Fig. 1. The crystalline SiC Y junction formed by the coalescence of two parallel branches follows the original $<111>\mathrm{SiC}$ growth direction. The merged catalyst yields a shared planar interface and creates a single crystal stem. However, for the crystalline $\mathrm{SiC}$ Y-junction formed by the coalescence of two inclined branches, the interface between the merged catalyst and stem is faceted and the stem is a bicrystal with a planar grain boundary along the center of the stem, where the bicrystal orientations reflect the orientation of each inclined branch. In contrast to the parallel-branch Y-junction where the growth direction stays along $<111>\mathrm{SiC}$, the inclined $\mathrm{Y}$-branch structure changes growth directions, which is similar to a previous report of Si nanowires where the $<111>$ and $<11-1>$ directions combined to yield a growth axes of $\langle 110>$. For both cases, the $\{111\} \mathrm{SiC}$ plane is formed at the metal droplet/SiC interface before and after coalescence of the metal droplets. At this interface the newly formed $\mathrm{SiC}$ follows the original crystal orientation resulting in epitaxial growth, supporting previous HRTEM studies that suggested the growth of the nanowire at the solid-liquid interface follows layer-by-layer stacking. The coalescence of the catalyst nanoparticles is driven by lowering the total surface energy; a similar mechanism was reported for the junction formation from carbon nanotubes and non-crystalline boron nanowires. The observation of crystalline SiC Y junctions with two parallel branches of diameter differences provides an insight into the dependence of the nanowire's growth kinetics on the catalyst size. Nanowire's growth kinetics related to different catalyst size and growth rate differences are caused by the Gibbs-Thomson or surface tension effect In situ TEM observations of the Fe atoms/clusters migration and agglomeration support the $\mathrm{SiC}$ nanocone or Y-junction formation. 
References:

[1] L. Manna, D. J. Milliron, A. Meisel, E. C. Scher, and A. P Alivisatos, Nature Materials 2, 382-385(2003).

[2] M. Sumper, S. Lorenz, and E. Brunner, Angew. Chem. Int. Ed. 42, 5192-5195(2003).

[3] Z. Liu, V. Srot, J. Yang, Applied Physics Letters, 96, 253111(2010).

[4] This research was supported in part by a grant from the NSF (DMR-0804892). We thank the Peterson Institute of Nanoscience and Engineering at University of Pittsburgh for facility.

a

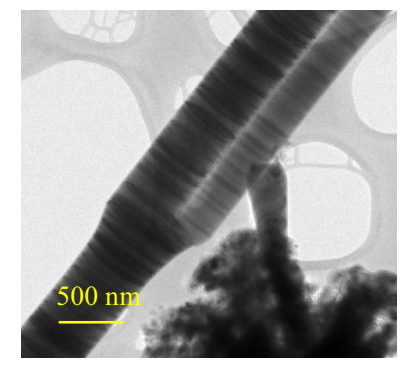

III<smiles>[Ge]C1CCCC1I</smiles>

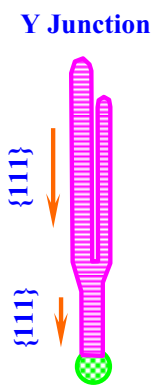

$\mathrm{b}$

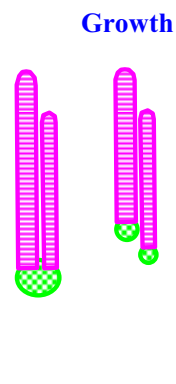

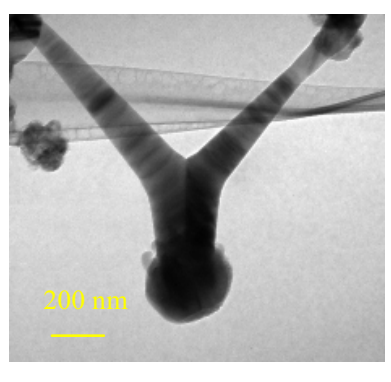

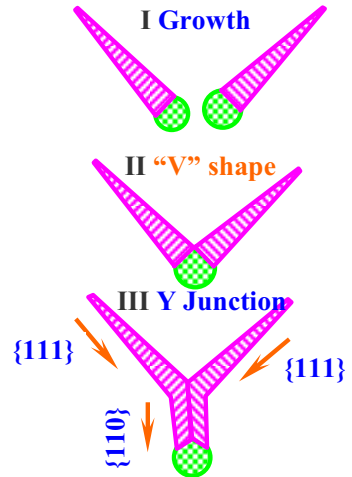

FIG. 1. TEM images and schematic diagram of the self-assembled crystalline SiC Y junctions created by the coalescence of two catalyst droplets: (a) Y junction with two parallel branches that merge into a single-crystal stem that follows the original growth direction; (b) Y junction with two inclined branches to form a stem with a planar grain boundary along the stem center and corresponding change of the growth direction.
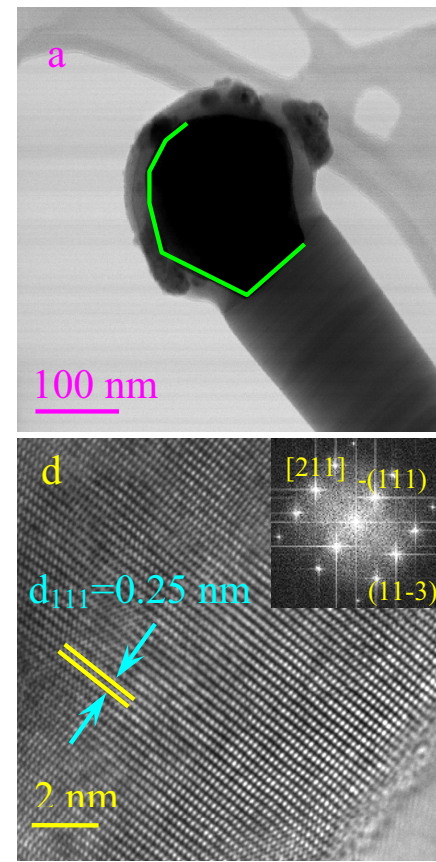

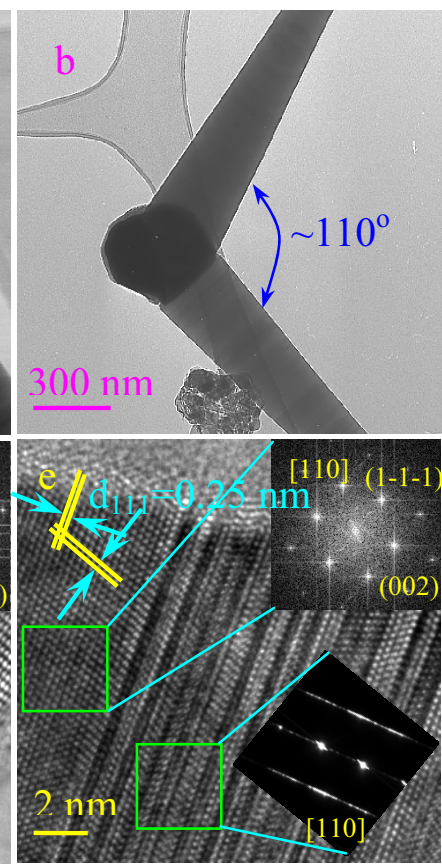

FIG. 2 Bright field (BF) TEM images of the interface between the catalysts and the nanocones (a-c). (a) A BF TEM image of a typical SiC-Fe interface; the line highlights the facet feature of the catalyst particle. (b) "V"-shape junction with two branches (interfaces) at a relative separation angle of $\sim 110^{\circ}$. (c) A BF TEM image of the stem portion of a $\mathrm{SiC} \mathrm{Y} \mathrm{junction,} \mathrm{showing} \mathrm{the} \mathrm{bicrystal} \mathrm{structure} \mathrm{of} \mathrm{the} \mathrm{stem} \mathrm{and} \mathrm{its} \mathrm{catalyst} \mathrm{droplet} \mathrm{with} \mathrm{a} \mathrm{faceted}$ interface, in which each facet is parallel to the $\{111\}$ planes of the bicrystal; the line highlights the interface. HRTEM images of the crystalline $\mathrm{SiC}$ Y junction branches along different projection directions (d) [211] and (e) [110]; the insets are the corresponding fast Fourier transformed images. (f) HRTEM image of the stem of a $\mathrm{SiC} \mathrm{Y}$ junction with two inclined branches, showing a planar grain boundary along the center. 